\title{
Design of two-dimensional PR quincunx filter banks with Euler- Frobenius polynomial and lifting scheme
}

\author{
MUKUND B NAGARE ${ }^{1}$, BHUSHAN D PATIL $^{2, *}$ and RAGHUNATH S HOLAMBE ${ }^{2}$ \\ ${ }^{1}$ Department of Instrumentation Engineering, Ramrao Adik Institute of Technology, Navi Mumbai, India \\ ${ }^{2}$ Department of Instrumentation Engineering, SGGS Institute of Engineering and Technology, Nanded, India \\ e-mail: nagaremukund@gmail.com; bhushandpatil@gmail.com; rsholambe@gmail.com
}

MS received 22 January 2019; revised 11 April 2019; accepted 16 April 2019; published online 18 June 2019

\begin{abstract}
Two-dimensional (2-D) filter banks (FBs) have played a significant role in retrieving the directional information of images. In this paper, we propose a technique to design 2-D two-channel perfect reconstruction (PR) FBs with quincunx sampling. The proposed design method comprises two stages. In the first stage, we propose the design of a new halfband polynomial using Euler-Frobenius polynomial $(E F P)$. This is constructed by imposing vanishing moment and PR constraints on $E F P$. The resulting new polynomial is a maximally flat Euler-Frobenius halfband polynomial $(E F H B P)$. Later, in the second stage, EFHBP is used in a modified 2-D lifting scheme to design 2-D filters. The design examples for 2-D filters are presented and compared to existing filters. The performance shows that proposed filters have better regularity, symmetry and less energy of the error compared with existing FBs. Finally, performance of designed filters is evaluated in image denoising application.
\end{abstract}

Keywords. Euler-Frobenius polynomial; 2-D quincunx filter banks; perfect reconstruction.

\section{Introduction}

Finding the efficient representation of images is a crucial challenge in all image processing applications, including feature extraction, denoising, compression, classification, etc [1]. The efficiency of presentation implies the ability to extract significant information of an image using small description. Wavelet filter banks (FBs) have turned out to be a very powerful tool for such applications. Wavelet filters should satisfy the properties such as perfect reconstruction (PR), linear phase, better frequency selectivity and desired vanishing moment (VM). To design nonseparable 2-D FBs including all aforementioned properties is a challenging task.

One-dimensional (1-D) wavelet FBs provides optimal representation for 1-D piecewise smooth signals [2, 3]. However, they fail to explore the geometric regularity that exists in majority of natural images and capture limited directional information. The directional information is an important feature of 2-D signals. To overcome these issues, many researchers have proposed 2-D nonseparable FBs design techniques, such as, directional FBs [1], contourlets [2], TIDFB [4] and 2-D quincunx FBs [5, 6]. These FBs extract the directional information of the natural images more efficiently. These designs are based on the McClellan transformation technique [1]. The limitation of these

*For correspondence methods is that we cannot explicitly control the shape of a frequency response of 2-D filters and filters do not achieve complete PR.

In this work, we design 2-D two-channel PR quincunx FBs using Euler-Frobenius polynomial EFP and lifting scheme. First, we design a new halfband polynomial using $E F P$. The resulting new polynomial is also known as a maximally flat Euler-Frobenius halfband polynomial $(E F H B P)$. This is constructed by imposing VMs and PR constraints on $E F P$. The $E F H B P$ proposed in the first stage is used in a modified 2-D lifting structure to design 2-D analysis low-pass and high-pass filters.

The proposed filters have better results as compared with existing FBs.

\section{Preliminaries}

\subsection{2-D two-channel quincunx $F B$}

The 2-D two-channel quincunx FBs structure is shown in figure 1 . The set of filters $H_{0}\left(z_{1}, z_{2}\right)$ and $H_{1}\left(z_{1}, z_{2}\right)$ represents the analysis of low-pass and high-pass filters, respectively. Similarly, $F_{0}\left(z_{1}, z_{2}\right)$ and $F_{1}\left(z_{1}, z_{2}\right)$ denote the synthesis filters. In these FBs, the nonseparable quincunx sampling $\quad \mathbf{D}=\left[\begin{array}{cc}1 & 1 \\ 1 & -1\end{array}\right]$ is used, which has down- 


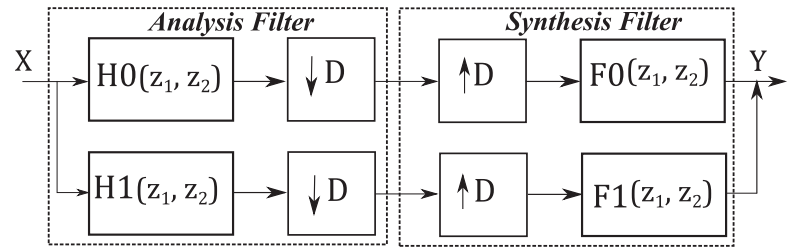

Figure 1. 2-D quincunx filterbanks with PR.

sampling ratio 2, i.e., $\operatorname{det}(\mathbf{D})=2$. The PR condition of 2-D FBs can be given as

$$
H_{0}\left(z_{1}, z_{2}\right) F_{0}\left(z_{1}, z_{2}\right)+H_{1}\left(z_{1}, z_{2}\right) F_{1}\left(z_{1}, z_{2}\right)=2 z_{1}^{-d_{1}} z_{2}^{-d_{2}}
$$

where $d_{1}$ and $d_{2}$ represent delays. The alias cancellation condition can be given as

$$
H_{0}\left(-z_{1},-z_{2}\right) F_{0}\left(z_{1}, z_{2}\right)+H_{1}\left(-z_{1},-z_{2}\right) F_{1}\left(z_{1}, z_{2}\right)=0 \text {. }
$$

Equation (2) is satisfied if we choose following filters:

$H_{1}\left(z_{1}, z_{2}\right)=-F_{0}\left(-z_{1},-z_{2}\right)$ and $F_{1}\left(z_{1}, z_{2}\right)=H_{0}\left(-z_{1},-z_{2}\right)$.

From Eq. (1), the product filter $P\left(z_{1}, z_{2}\right)$ can be defined as $P\left(z_{1}, z_{2}\right)=H_{0}\left(z_{1}, z_{2}\right) F_{0}\left(z_{1}, z_{2}\right)$, which gives

$$
P\left(z_{1}, z_{2}\right)+P\left(-z_{1},-z_{2}\right)=2 z_{1}^{-d_{1}} z_{2}^{-d_{2}} .
$$

Hence, the aim of designing 2-D directional FBs with PR condition is the problem of designing $P\left(z_{1}, z_{2}\right)$ by imposing the respective constraints.

\section{Proposed method to design quincunx FB}

In this section, a method to design 2-D two-channel PR FBs with quincunx sampling is proposed.

\subsection{Design of new halfband polynomial}

The EFP is well known and has been used in the design of spline wavelets [3]. The EFP has the property that its coefficients are integers and the polynomial is symmetric. These coefficients are determined recursively. The generalized $E F P$ is defined as

$$
\mathcal{E}_{f}(z)=\sum_{i=0}^{m} \hat{e}(i+1) z^{-i}
$$

where $m$ represents the order of $E F P$ and its coefficients $(\hat{e})$ are obtained as

$$
\hat{e}(i+1)=\sum_{\ell=0}^{i}(-1)^{\ell}\left(\begin{array}{c}
m+2 \\
\ell
\end{array}\right)(i+1-\ell)^{m+1} .
$$

In this work, we have used EFPs to design a new maximally flat halfband polynomial. The odd-length EFPs are combined with VMs (zeros at $z=-1$ ) and some independent parameters $\beta_{i}$ are introduced so as to convert them into halfband polynomials. This new polynomial is called as $E F H B P$ and is given as

$$
P(z)=\left(1+z^{-1}\right)^{\eta} \mathcal{E}_{f}(z) \sum_{i=0}^{L} \beta_{i} z^{-i}
$$

where $\eta$ represents the number of VMs. The $m$ th-order $E F P$ is represented by $\mathcal{E}_{f}(z)$. The product filter $P(z)$ has order $\kappa=\eta+m+L$. The order of free polynomial is $L=\kappa / 2-1$. The free parameters $\beta_{i}$ are formulated such that the PR condition given in Eq. (1) is satisfied. We get different halfband filters by changing the degree of VMs and order of EFP. We consider Eq. (6) to design the 2-D lifting kernels.

\subsection{Modified 2-D lifting structure}

The proposed 2-D filters are constructed via modified 2-D lifting structure as shown in figure 2 . We have considered the three lifting steps to design 2-D FBs. The analysis FBs can be represented in polyphase matrix form as

$$
\mathbf{P}=\left[\begin{array}{ll}
H_{0 e v e n} & H_{0 o d d} \\
H_{1 \text { even }} & H_{1 \text { odd }}
\end{array}\right] .
$$

From the polyphase matrix, we can find the filters $H_{0}$ and $H_{1}$ as

$$
\begin{aligned}
& H_{0}\left(z_{1}, z_{2}\right)=H_{\text {0even }}\left(z_{1}^{2}, z_{2}^{2}\right)+z_{1}^{-1} z_{2}^{-1} H_{0 \text { odd }}\left(z_{1}^{2}, z_{2}^{2}\right), \\
& H_{1}\left(z_{1}, z_{2}\right)=H_{\text {leven }}\left(z_{1}^{2}, z_{2}^{2}\right)+z_{1}^{-1} z_{2}^{-1} H_{1 \text { odd }}\left(z_{1}^{2}, z_{2}^{2}\right) .
\end{aligned}
$$

The inverse of polyphase matrix $\mathbf{P}$ represents the synthesis polyphase matrix $\mathbf{S}=\mathbf{P}^{-1}$. To satisfy the PR property, we should have $\mathbf{P} . \mathbf{S}=I$. The general prediction step can be obtained by multiplication with matrix of the form $P=$ $\left[\begin{array}{cc}1 & 0 \\ -p_{0} P_{1}(z) & 1\end{array}\right]$ and update step is given by $U=\left[\begin{array}{cc}1 & p_{1} U_{1}(z) \\ 0 & 1\end{array}\right]$. The polyphase representation for three-step lifting is given by

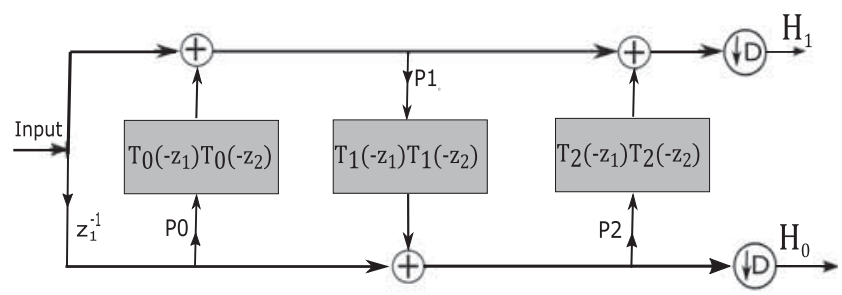

Figure 2. Modified 2-D lifting structure. 

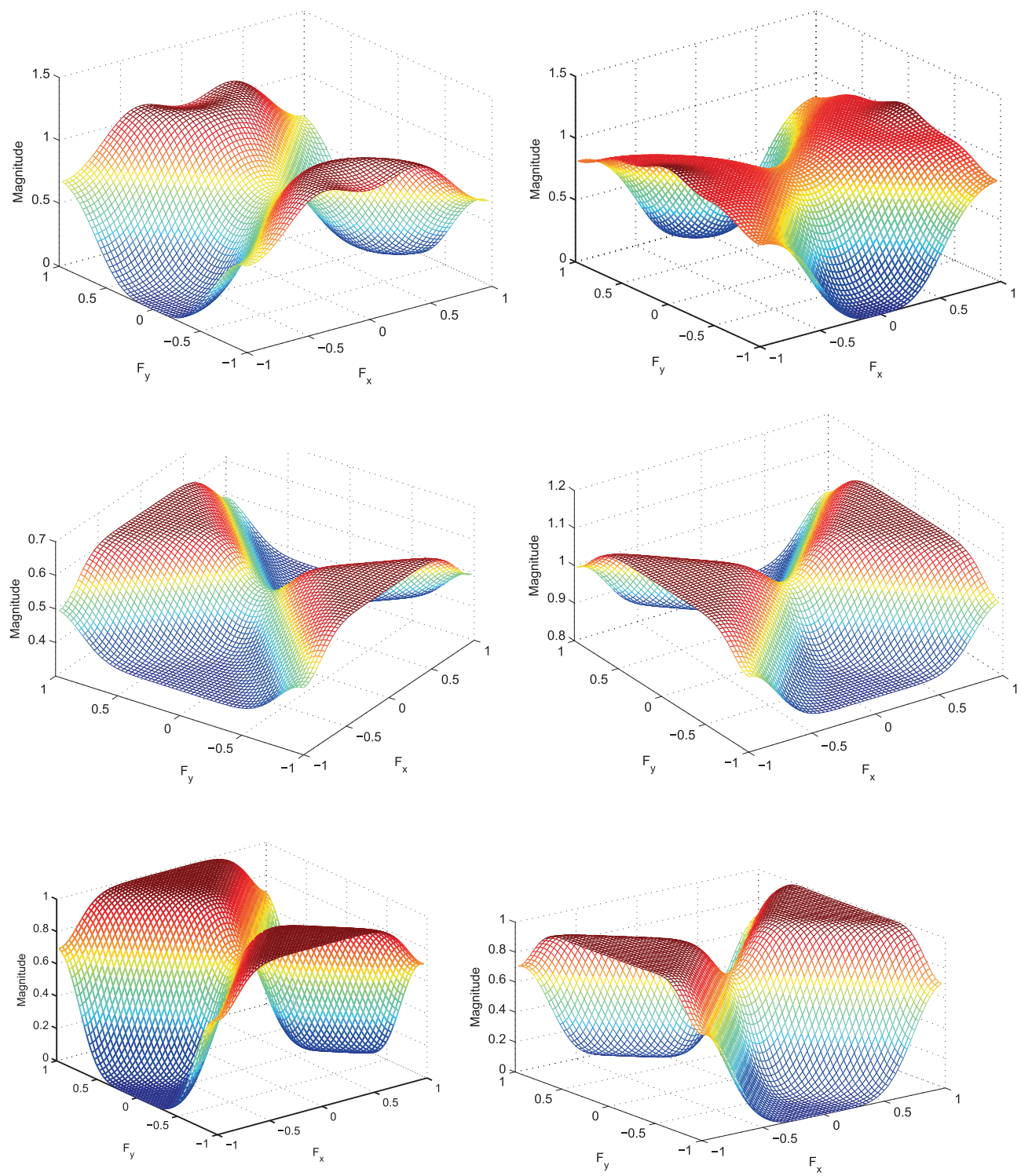

Figure 3. First row: Fan filters designed with McClellan transformation [1]. Second row: Eslami's method [4]. Third row: Proposed method.

$\mathbf{P}=\left[\begin{array}{cc}1 & 0 \\ -p_{0} P_{1}\left(z_{1}, z_{2}\right) & 1\end{array}\right]\left[\begin{array}{cc}1 & p_{1} U_{1}\left(z_{1}, z_{2}\right) \\ 0 & 1\end{array}\right]\left[\begin{array}{cc}1 & 0 \\ -p_{2} P_{2}\left(z_{1}, z_{2}\right) & 1\end{array}\right]$

where $p_{0}, p_{1}$ and $p_{2}$ are non-zero lifting parameters. The prediction and update kernels are designed from $\kappa^{\text {th }}$-order halfband polynomials, let us say $\mathcal{T}_{i}(z)$, and obtained as

$$
P_{i}=U_{i}=\mathcal{T}_{i}=z^{\kappa / 2} P(z)-1 \quad \text { where } \quad i=0,1,2 .
$$

In the proposed method, we map 1-D halfband polynomialbased kernels into 2-D using a transformation on the three halfband lifting kernels $\mathcal{T}_{0}(z), \mathcal{T}_{1}(z)$ and $\mathcal{T}_{2}(z)$. We replace all the $1-\mathrm{D}$ kernels $\mathcal{T}_{i}(z)$ with $2-\mathrm{D}$ halfband kernels as

$$
\mathcal{T}_{i}(z)=\mathcal{T}_{i}\left(-z_{1}\right) \mathcal{T}_{i}\left(-z_{2}\right) \quad \text { where } i=0,1,2
$$

Subsequently, the 1-D delay $\left(z^{-1}\right)$ that occurs is replaced as 2-D delay $z_{1}^{-1} z_{2}^{-1}$. Using this transformation in lifting as shown in figure 2, the 2-D analysis low-pass and synthesis low-pass filters are expressed as

$H_{0}=\frac{1+p}{2}+\frac{1}{2} \mathcal{T}_{1}\left(-z_{1}\right) \mathcal{T}_{1}\left(-z_{2}\right)\left[1-p \mathcal{T}_{0}\left(-z_{1}\right) \mathcal{T}_{0}\left(-z_{2}\right)\right]$

$$
\begin{aligned}
F_{0}= & \frac{1+p \mathcal{T}_{0}\left(-z_{1}\right) \mathcal{T}_{0}\left(-z_{2}\right)}{1+p}+\frac{1-p}{1+p} \mathcal{T}_{2}\left(-z_{1}\right) \mathcal{T}_{2}\left(-z_{2}\right) \\
& \times\left\{\frac{1+p}{2}-\frac{1}{2} \mathcal{T}_{1}\left(-z_{1}\right) \mathcal{T}_{1}\left(-z_{2}\right)\left[1+p \mathcal{T}_{0}\left(-z_{1}\right) \mathcal{T}_{0}\left(-z_{2}\right)\right]\right\}
\end{aligned}
$$

where the kernels $\mathcal{T}_{i}(z)$ for $i=0,1,2$ are designed from $\kappa^{\text {th }}$-order proposed halfband polynomials $P(z)$. The 

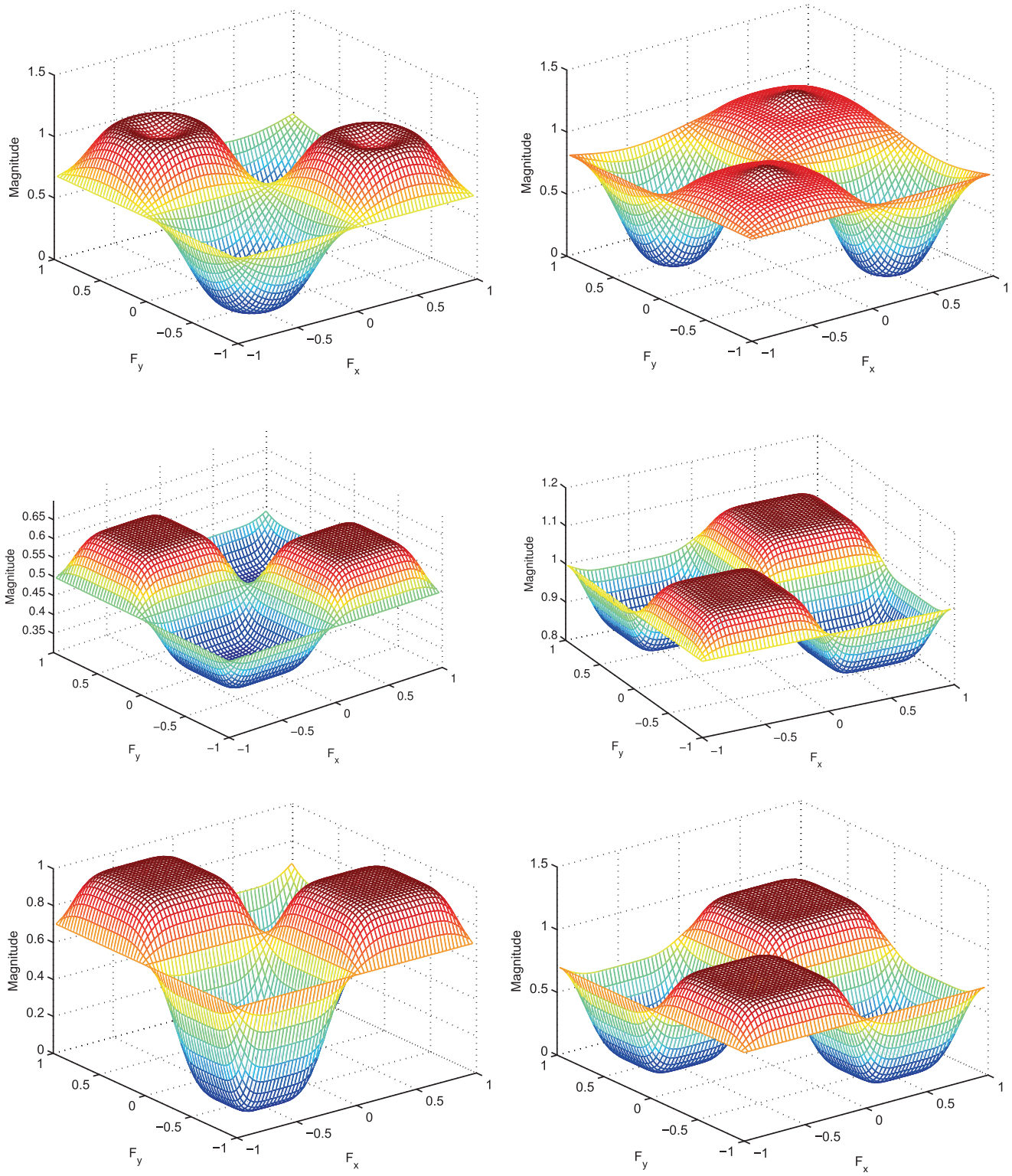

Figure 4. First row: Checkerboard filters designed with McClellan transformation [1]. Second row: Eslami's Method [4]. Third row: Proposed method.

objective is to design these kernels such that the resultant 2-D analysis and synthesis filters have good frequency selectivity and desired regularity. The 2-D analysis and synthesis high-pass filters have been constructed using Eq. (2). The lifting parameter $p$ is the degree of freedom that provides the flexibility for choosing the magnitude at $\omega=\pi / 2$.

\subsection{Choice of lifting parameters}

Note that the filters and kernels obtained from aforementioned equations are non-causal filters. Therefore, by applying $\kappa / 2$ delays to the product filter we get causal kernels as given in Eq. (11). The parameters $p_{0}, p_{1}$ and $p_{2}$ are determined by examining the passband and stopband response of the 2-D analysis low-pass filter and high-pass filter as $p_{0}=p_{1}=(1+p) / 2 \quad$ and $p_{2}=(1-p) /(1+p)$. The free parameters $p_{i}$ are used to provide flexibility for shaping the frequency response of designed filters and its value is set in the range of $0<p<1$. With the appropriate value of $p$, we can set the frequency response of designed filters to any desired value at $\omega=\pi / 2$. Particularly, we select $p=\sqrt{2}-1$ and frequency response magnitude at $\pi / 2$ is $1 / \sqrt{2}$ for both $H_{0}$ and $F_{0}$ (see [1]). 
Table 1. Properties of the designed 2-D filters.

\begin{tabular}{llccr}
\hline Filter & \multicolumn{1}{c}{ Method } & Energy of the error & Symmetry & Regularity \\
\hline Fan & Eslami and Radha [4] & $10.0611 \mathrm{e}+05$ & 0.0135 & 0.0608 \\
& Rahulkar and Holambe [1] & $1.3434 \mathrm{e}+06$ & 1.5931 & -0.18616 \\
& Proposed & $5.7977 \mathrm{e}+05$ & 0.0135 & 0.0069 \\
Checkerboard & Eslami and Radha [4] & $2.4596 \mathrm{e}+05$ & 0.89462 & -0.05185 \\
& Rahulkar and Holambe [1] & $8.3711 \mathrm{e}+06$ & 1.53469 & 0.19078 \\
& Proposed & $8.2650 \mathrm{e}+05$ & 0.0188 & 0.0056 \\
\hline
\end{tabular}

\section{Design examples}

This section illustrates a few design examples. We can obtain different halfband filters by choosing different VMs, $\eta=1,2,3$ and order $m$ of EFP in Eq. (6). Here, we have considered a fan-shaped and checkerboard-shaped 2-D filters.

\subsection{Construction of 2-D fan shape FBs}

In this example, the $10^{\text {th }}$-order $E F H B P$ is used to design $21 \times 21$ size fan shape filters. The five independent parameters are imposed as a PR constraint by selecting $\ell=4$ to obtain halfband filters. If we select $\eta=4$ in order to achieve maximum regularity in designed filters, then halfband PR condition yields five linear equations:

$$
\begin{gathered}
\beta_{3}+6 \beta_{4}=0, \\
20 \beta_{4}+15 \beta_{3}+6 \beta_{2}+\beta_{1}=0, \\
6 \beta_{4}+15 \beta_{3}+20 \beta_{2}+15 \beta_{1}+6 \beta_{0}=1, \\
\beta_{3}+6 \beta_{2}+15 \beta_{1}+\beta_{0}=0,
\end{gathered}
$$

$$
\beta_{1}+6 \beta_{0}=0 .
$$

On solving this set of linear equations, we get independent parameters as $\beta_{0}=-0.01171875, \beta_{1}=-0.0703125$,

$\beta_{2}=-0.1484375, \quad \beta_{3}=-0.0703125 \quad$ and $\beta_{4}=-0.01171875$. Putting these values in Eq. (6), we get the resultant halfband polynomial $P(z)$. Next, 2-D fanshaped filters are designed by selecting identical kernels $\mathcal{T}_{i}\left(-z_{1}\right) \mathcal{T}_{i}\left(-z_{2}\right)$ in modified lifting structure. When all kernels are identical, then

$$
\begin{aligned}
\mathcal{T}_{i}\left(-z_{1}\right) \mathcal{T}_{i}\left(-z_{2}\right) & =\mathcal{T}_{0}\left(-z_{1}\right) \mathcal{T}_{0}\left(-z_{2}\right)=\mathcal{T}_{1}\left(-z_{1}\right) \mathcal{T}_{1}\left(-z_{2}\right) \\
& =\mathcal{T}_{2}\left(-z_{1}\right) \mathcal{T}_{2}\left(-z_{2}\right)=z_{1}^{-\kappa / 2} z_{2}^{-\kappa / 2} P\left(z_{1}\right) P\left(z_{2}\right)-1 .
\end{aligned}
$$

These kernels are substituted in (13) and (14) to obtain 2-D fan-shaped analysis and synthesis low-pass filters. The analysis low-pass filter $h_{0}\left(n_{1}, n_{2}\right)$ is a quadrantal-symmetric filter and its second quadrant coefficients are given in Eq. (20), where its centre value is 0.6038. Also, the synthesis low-pass filter $g_{0}\left(n_{1}, n_{2}\right)$ is a quadrantal-symmetric filter and its second quadrant coefficients are given in Eq. (21). The frequency response of designed filters is shown in figure 3.

$$
\begin{aligned}
& h_{0}\left(n_{1}, n_{2}\right)= \\
& {\left[\begin{array}{cccccccccccccc}
0 & 0 & 0 & 0 & 0 & 0 & 0 & 0 & 0 & 0 \\
0 & 0 & 0 & 0 & 0 & 0 & 0 & 0 & 0 & -6.5 \mathrm{e}-08 & 0 \\
0 & 0 & 0 & 0 & 0 & 0 & 0 & 0 & -6.6 \mathrm{e}-07 & 0 & -1.0 \mathrm{e}-06 \\
0 & 0 & 0 & 0 & 0 & 0 & 0 & -2.8 \mathrm{e}-06 & 0 & -1.1 \mathrm{e}-05 & 0 \\
0 & 0 & 0 & 0 & 0 & 0 & -6.4 \mathrm{e}-06 & 0 & -4.7 \mathrm{e}-05 & 0 & -0.0001 \\
0 & 0 & 0 & 0 & 0 & 2.0 \mathrm{e}-05 & 0 & -0.0001 & 0 & -0.0004 & -6.8 \mathrm{e}-05 \\
0 & 0 & 0 & 0 & -6.4 \mathrm{e}-06 & 0 & 0.0003 & 0 & -0.001 & -0.0005 & -0.002 \\
0 & 0 & 0 & -2.8 \mathrm{e}-06 & 0 & -0.0001 & 0 & 0.003 & -0.003 & -0.004 & -0.004 \\
0 & 0 & -6.6 \mathrm{e}-07 & 0 & -4.7 \mathrm{e}-05 & 0 & -0.001 & 0.003 & 0.01 & -0.02 & -0.01 \\
0 & -6.5 \mathrm{e}-08 & 0 & -1.1 \mathrm{e}-05 & 0 & -0.0004 & 0.0005 & -0.004 & 0.02 & 0.03 & -0.17 \\
-3.9 \mathrm{e}-09 & 0 & -1.0 \mathrm{e}-06 & 0 & -0.0001 & 6.8 \mathrm{e}-05 & -0.002 & 0.004 & -0.01 & 0.17 & 0.6038
\end{array}\right]}
\end{aligned}
$$




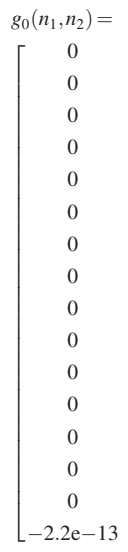

\subsection{Construction of 2-D checkerboard-shaped FBs}

The fan shape filters designed by proposed method are down-sampled by quincunx sampling $D$ to design the checkerboard-shaped filters; for example

$$
\begin{aligned}
& H_{0}^{c h}\left(z_{1}, z_{2}\right)=H_{0}^{f}\left(z_{1} z_{2}, z_{1} z_{2}^{-1}\right) . \\
& F_{0}^{c h}\left(z_{1}, z_{2}\right)=F_{0}^{f}\left(z_{1} z_{2}, z_{1} z_{2}^{-1}\right) .
\end{aligned}
$$

We have used same design parameters in the checkerboard shape filter design as fan-shaped filters. The frequency responses of analysis low-pass $H_{0}^{c h}\left(w_{1}, w_{2}\right)$ and synthesis low-pass $F_{0}^{c h}\left(w_{1}, w_{2}\right)$ filters are shown in figure 4 .

Properties such as regularity, symmetry and frequency selectivity are measured for proposed filters and compared to existing methods as given in table 1. Using Eq. (1), we have ensured that the proposed filters satisfy PR condition with value equal to 1 . The performance is not degraded for the filters such as $21 \times 21,23 \times 23,27 \times 27$ and $29 \times 29$ size 2-D filters. However, complexity may increase for higher length filters. The higher length filters can be designed using either higher-order EFHBP or additional lifting steps. We have compared the proposed fan-shaped and checkerboard-shaped 2-D filters to the filters designed by the McClellan transformation method [1] and filters given in [4]. The comparisons of the frequency responses are shown in figures 3 and 4 . Although complexity is high for higher length filters, the filters yield better frequency response than that from the McClellan transformation method [1] and filters given in [4].

\section{Application to image denoising}

To illustrate the effectiveness of the proposed filters, we have carried out experiments on image denoising application. The proposed filters are used in denoising scheme (TIDFB) [4] as shown in figure 5. We have tested performance of designed 2-D filters on the standard Barbara $(512 \times 512)$ image. The test image is contaminated

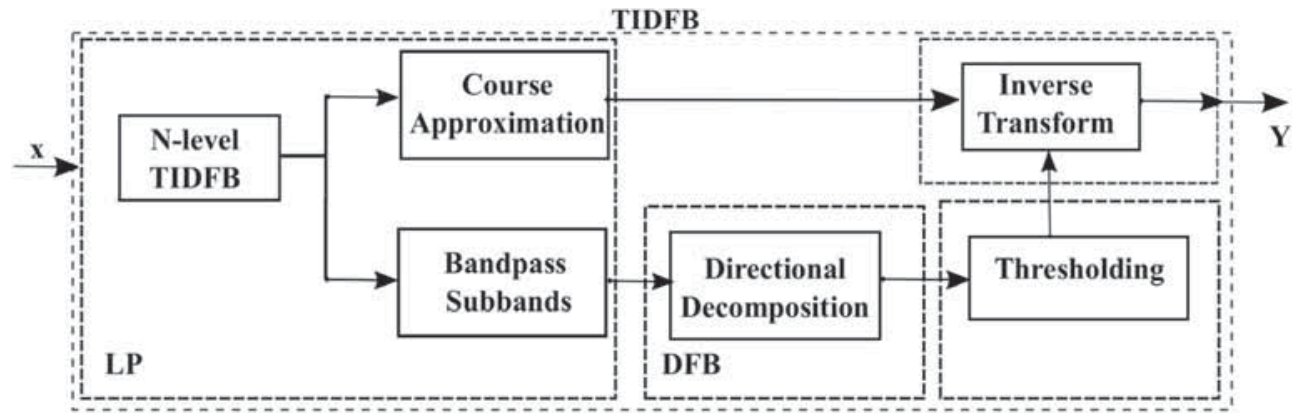

Figure 5. Denoising scheme using proposed filters. 
Table 2. Result of denoising experiments (PSNR (dB)).

\begin{tabular}{lccccc}
\hline Sigma & Noisy image & CT [2] & TIWT [4] & TICT [4] & Proposed \\
\hline 10 & 28.13 & 29.27 & 32.52 & 33.46 & 34.06 \\
20 & 22.11 & 26.027 & 28.22 & 29.48 & 30.08 \\
30 & 18.57 & 24.25 & 25.89 & 27.26 & 28.86 \\
40 & 16.08 & 23.16 & 24.50 & 25.68 & 27.28 \\
50 & 14.13 & 22.24 & 23.58 & 24.59 & 25.78 \\
60 & 12.56 & 21.65 & 22.83 & 23.60 & 24.02 \\
\hline
\end{tabular}

first by adding a zero-mean Gaussian white noise with a standard deviation of $\sigma$. For all the denoising schemes, we assumed that $\sigma$ is unknown and we estimated it using the robust median estimator as given in [4]. These noisy images are then used in the denoising scheme. The denoising scheme mainly consists of two stages: a Laplacian Pyramid (LP) decomposition [2] and directional filter bank (DFB) decomposition. The proposed filters are used in directional decomposition as shown in figure 5. The LP decomposes the standard images (e.g. Barbara) into approximation information and radial bandpass subbands with different directional information. Later DFB is applied to each radial bandpass subband where maximum fine structural details of Barbara image for different directions are extracted efficiently. In these experiments, the images are decomposed up to three level directional decompositions. Noise generally contains high-frequency component, due to which it lies in the high-frequency subbands. Therefore, we threshold the detail subbands to suppress the noise content present in the image and reconstruct the original image. The noise is suppressed using hard thresholding and it is estimated using Bayes' shrinkage rule. The denoising performance of the proposed filters is compared to that of existing well-known methods, such as Contourlet transform (CT) [2], TIWT [4] and TICT [4]. In order to obtain an insightful analysis, the qualitative objective measure of peak signal to noise ratio (PSNR) is measured. Table 2 depicts the PSNR values of denoising results. The standard deviation of the input noise is considered between $\sigma=10$ and $\sigma=60$. From the results, it is observed that the proposed 2-D filters perform better in denoising and results are shown in figure 6.

\section{Conclusion}

In this paper, an efficient technique to design 2-D quincunx FBs with PR has been proposed. The modified 2-D lifting structure and EFHBP are used to construct a new class of 2-D fan- and checkerboard-shaped filters. The designed filters have satisfied PR property. The performance of the properties indicates that the proposed 2-D filters give better symmetry, regularity and frequency selectivity as compared with existing methods. The

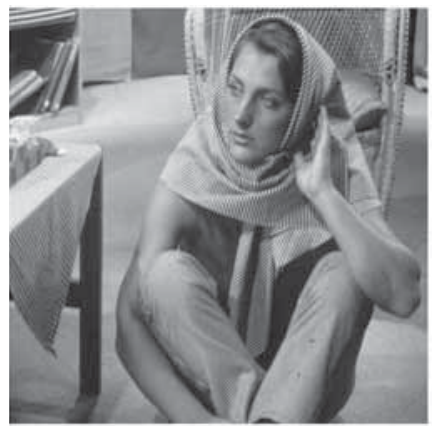

(a)

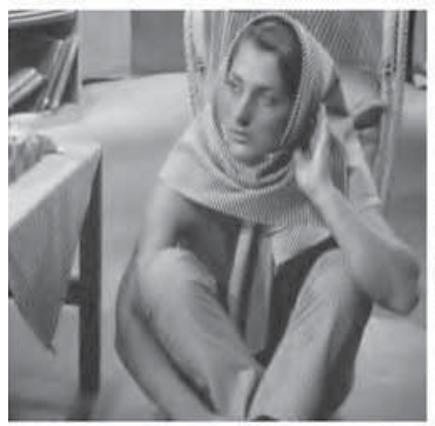

(d)

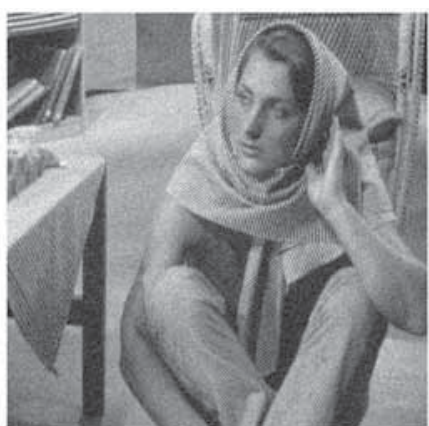

(b)

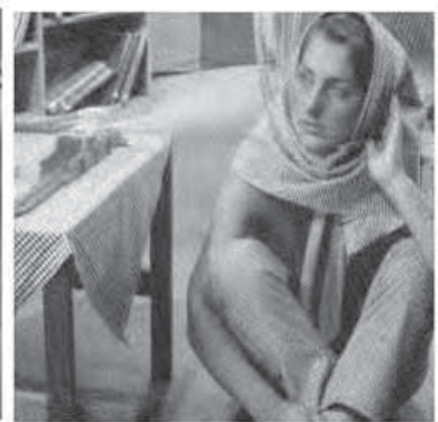

(e)

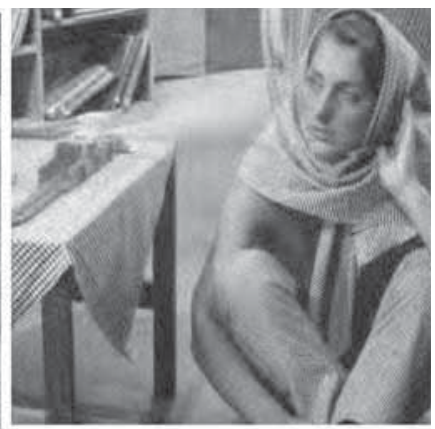

(c)

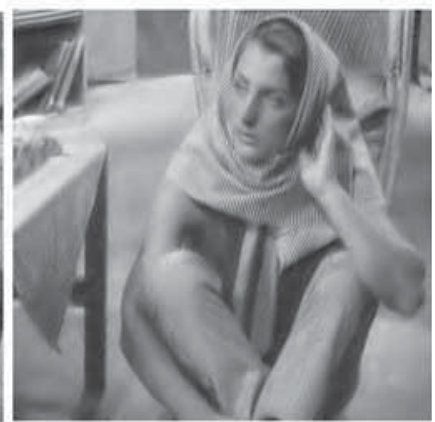

(f)

Figure 6. Denoising results: (a) original image, (b) noisy image $(\sigma=20)$, (c) CT [2], (d) TIWT [4], (e) TICT [4] and (f) proposed method. 
proposed method comparatively gives good denoising results over existing methods.

\section{References}

[1] Rahulkar A D and Holambe R S 2014 Iris image recognition. In: Wavelet Filter-banks Based Iris Feature Extraction Schemes, Springer Briefs in Signal Processing

[2] Do M N and Vetterli M 2005 The contourlet transform: an efficient directional multiresolution image representation. IEEE Trans. Image Process. 14: 2091-2106
[3] Gawande J P, Rahulkar A D and Holambe R S 2016 A new approach to design triplet halfband filter banks based on balanceduncertainty optimization. Digit. Signal Process. 56: 123-131

[4] Eslami R and Radha H 2006 Translation-invariant contourlet transform and its application to image denoising. IEEE Trans. Image Process. 15: 3362-3374

[5] Nagare M B, Patil B D and Holambe R S 2017 A multi directional perfect reconstruction filter bank designed with 2-D eigenfilter approach: application to ultrasound speckle reduction. J. Med. Syst. 41: 31

[6] Nagare M B, Patil B D and Holambe R S 2016 Design of twodimensional quincunx FIR filter banks using Eigen filter approach. In: Proceedings of the 2016 International Conference on Signal and Information Processing (IConSIP), pp. 1-5 